\title{
Thermal denaturation and folding rates of single domain proteins: size matters
}

\author{
Mai Suan $\mathrm{Li}^{1}$, D. K. Klimov ${ }^{2}$ and D. Thirumalai ${ }^{2}$ \\ ${ }^{1}$ Institute of Physics, Polish Academy of Sciences, Al. Lotnikow 32/46, 02-668 Warsaw, Poland \\ ${ }^{2}$ Institute for Physical Science and Technology, University of Maryland, College Park, MD 20742
}

\begin{abstract}
We analyze the dependence of thermal denaturation transition and folding rates of globular proteins on the number of amino acid residues, $N$. Using lattice Go models we show that $\Delta T / T_{F} \sim N^{-1}$, where $T_{F}$ is the folding transition temperature and $\Delta T$ is the transition width computed using the temperature dependence of the order parameter that distinguishes between the unfolded state and the native basin of attraction. This finding is consistent with finite size effects expected for the systems undergoing a phase transition from a disordered to an ordered phase. The dependence of the folding rates on $N$ for lattice models and the dataset of 57 proteins and peptides shows that $k_{F} \simeq k_{F}^{0} \exp \left(-C N^{\beta}\right)$ with $0<\beta \leq 2 / 3$ provides a good fit, where $C$ is a $\beta$-dependent constant. We find that $k_{F} \simeq k_{F}^{0} \exp \left(-1.1 N^{\frac{1}{2}}\right)$ with an average (over the dataset of proteins) $k_{F}^{0} \approx(0.4 \mu s)^{-1}$, can estimate optimal protein folding rates, to within an order of magnitude in most cases. By using this fit for a set of proteins with $\beta$-sheet topology we find that $k_{F}^{0} \approx k_{U}^{0}$, the prefactor for unfolding. The maximum ratio of $k_{U}^{0} / k_{F}^{0} \approx 10$ for this class of proteins.
\end{abstract}

\section{INTRODUCTION}

Deciphering the factors that determine the foldability of protein sequences [1, 2, 3, 4, 4, 5] is an important problem from the perspective of protein design, protein structure prediction, and in vitro and in vivo protein folding. Foldability refers both to the folding rate, $k_{F}$, and thermodynamics of the transition from the ensemble of unfolded states $(\mathbf{U})$ to the native state or, more precisely, to the native basin of attraction (NBA). Folding rates and the associated equilibrium characteristics depend on intrinsic factors (sequence and topology) as well as on external conditions ( $\mathrm{pH}$, temperature, salt concentration, and viscosity). Variation in external conditions can not only alter the rates, but also the mechanism of folding. Despite this obvious fact most of the studies have been focused on the dependence of $k_{F}$ solely on the characteristics of the native states as described by the crystal (or NMR) structures.

The role of finite size effects on the thermodynamics of protein folding has received very little attention. The emphasis on the cooperativity of the transition from $\mathbf{U}$ state to NBA seems to have precluded consideration of the role of $N$, the number of residues in a sequence. This transition, for apparent two-state folders, has all the hallmarks of (weak) first-order phase transition. The highly cooperative $\mathbf{U} \leftrightarrow \mathbf{N B A}$ transition has lead some 
authors to suggest that there is no evidence that partially structured states contribute to the thermodynamic properties of proteins. Computational studies have shown [6] that in $\beta$-hairpin forming sequence from the C-terminus of GB1 protein structure is acquired over a finite range of temperatures, even though the overall folding can be described as a broad "two-state" transition [7]. Experiments on refolding of barnase have also suggested that structure is lost incrementally upon temperature induced unfolding [8]. Direct temperature dependence of structure formation in leucine zipper using one dimensional NMR experiments has established that melting temperature varies across the structure [9]. Although the variations occur over a relatively narrow range of temperatures, it is clear from these experiments that because of the finite size of proteins partially folded structures contribute to folding thermodynamics. These observations warrant an examination of finite size effect on the $\mathbf{U} \leftrightarrow$ NBA transition. Building on our previous study [10], we further investigate the role of $N$ in thermal denaturation using lattice models of proteins.

It has been noted [11] that $k_{F}$ correlates well with the relative contact order (RCO), which measures the proximity of side chain contacts in the folded state. The notion that protein folding is initiated with residues forming local structures and, thus, is determined by their proximity along the sequence constitutes the basis of the hierarchical folding mechanism [12]. Thus, in retrospect, the correlation between the RCO and $k_{F}$ is not entirely unanticipated, especially in $\alpha$-helical proteins. Although RCO is an important indicator of the folding rates, it should be pointed out that there is little correlation between RCO and $k_{F}$ for proteins with $\beta$-sheet topology. Clarke et al [13] showed that neither $k_{F}$ nor the unfolding rates $k_{U}$ correlate with RCO for a class of $\beta$-sheet proteins belonging to the immunoglobulin (Ig) fold. The RCO for the 6 proteins examined [13] is in the very narrow range $(0.17 \leq \mathrm{RCO} \leq 0.20)$. Nevertheless, the refolding rates for these proteins vary by a factor of 800 . More recently, Clarke and coworkers have shown that for a number of Ig domains from the muscle protein titin $k_{F}$ can vary by over four orders of magnitude [14, although their RCO values are expected to be nearly the same. These studies show that factors besides RCO play an important role in the determination of $k_{F}$.

Surprisingly, it was initially suggested [11] that neither stability nor the size of proteins plays a role in determining $k_{F}$. These counterintuitive observations contradict several theoretical [15, 16, 17, 18] and a few experimental studies [13. More careful examination of the database of well characterized proteins has shown that, although there are exceptions [19], stability is an important factor that determines $k_{F}$ [13,20]. Recently, several studies 21,22 have concluded that $N$, the number of amino acids, must also play an important role in determining $k_{F}$. In this paper we examine the dependence of rates as well as thermal denaturation of single domain proteins on $N$.

Beginning with the paper by one of us [15] a number of theoretical studies [16,17, 18] have predicted that $N$ should play a significant role in controlling $k_{F}$. Given that polypeptide chains are heteropolymers we expect that their relaxation times in both the folded and unfolded states must depend on $N$. Theoretical studies [15,16] suggest that the dependence of $k_{F}$ on $N$ is dictated by the interplay of three characteristic temperatures of the polypeptide chain, namely, $T_{F}$ (the folding transition temperature), $T_{\theta}$ (the collapse transition temperature), and $T_{g}$ (the glass transition temperature). It appears that in most experiments the external conditions are such that fastest folding is observed near the "tricritical" point, where $T_{F} \approx T_{\theta}$ in accord with the prediction by Camacho and Thirumalai [23]. For near 
optimal folding, as it may be the case for minimally frustrated sequences, it has been argued that

$$
\ln \left(k_{F} / k_{F}^{0}\right) \sim \alpha \ln N
$$

where $\alpha \approx 4$ [15] and $k_{F}^{0}$ is an undetermined prefactor. For artificial Go models $\alpha \approx 3$ [18,24]. On the other hand, due to topological frustration, even the sequences following two-state kinetics have a rough energy landscape. In this case

$$
\ln \left(k_{F} / k_{F}^{0}\right) \sim N^{\beta}
$$

The value of $\beta$ has been suggested to be less than unity and is probably in the range $0.5 \leq \beta \leq \frac{2}{3}$ 15.16.17. Given the limited range of $N$ for single domain proteins it is difficult (see below) to determine $\beta$ precisely.

To probe finite size effects on thermally induced folding we have performed Monte Carlo simulations using Go lattice models. These results are used to quantitatively establish the effect of finite $N$ on rounding the $\mathbf{U} \leftrightarrow$ NBA transition. A dataset of proteins, for which $k_{F}$ is available, is used to draw lessons on the dependence of $k_{F}$ on $N$. Using these results we show that unambiguous determination of $\beta$ is not possible. However, we argue that the $N$ dependence given in Eq. (2) is useful in analyzing the experimental data. As a byproduct of this work we also provide estimates of the folding and unfolding prefactors, $k_{F}^{0}$ and $k_{U}^{0}$.

\section{MODELS AND METHODS}

For the numerical simulations we represent a polypeptide chain using lattice Go model without side chains. The energy of a conformation

$$
E=\sum_{i<j} \epsilon_{i j} \delta_{r_{i j}, a}
$$

where $a$ is a lattice spacing, $r_{i j}$ is the distance between non-bonded beads $i$ and $j$, and the contact energies $\epsilon_{i j}$ are chosen to be -1 for native contacts and 0 for non-native ones. Go models are useful in exploring general physical principles that govern protein folding under the condition of marginal stability of the native state [25,26]. The sequences were selected by a standard sequence space Monte Carlo algorithm, which maximizes the Z-score for a given target structure. The target structures for each $N$ were chosen to be maximally compact. For example, for $N=18$ and $N=80$ the native structures occupy the vertexes of $3 \times 3 \times 2$ and $4 \times 4 \times 5$ cubes, respectively.

The thermodynamics of folding were determined using Monte Carlo simulations based on MS3 move set [27,28,29,30], which involves single, double and triple bead moves. Because this move set involves multiparticle updates, it is much more efficient compared to the standard move set 29,30,31]. The thermodynamic properties of the sequences are calculated using the multiple histogram method [32]. Typical number of Monte Carlo trajectories used to collect histograms is 50-100 depending on $N$. The free energy is calculated as a function of the number of native contacts $Q$, which is treated as an approximate reaction coordinate for Go models. This allows us to estimate the dependence of folding and unfolding free energy barriers on $N$. 
For lattice models the structural similarity with the native conformation is measured by the overlap function 23]

$$
\chi=1-\frac{1}{N^{2}-3 N+2} \sum_{i<j+1}^{N} \delta\left(r_{i j}-r_{i j}^{0}\right),
$$

where the superscript 0 refers to the native state. The folding temperature $T_{F}$ is defined as a temperature at which $d\langle\chi\rangle / d T$ is maximum and the transition width $\Delta T$ is defined as the full width at half maximum of $d<\chi>/ d T$ at $T=T_{F}$.

\section{RESULTS}

A. Finite size effects in thermal denaturation: The transition width $\Delta T$ is obtained from the temperature dependence of $d\langle\chi\rangle / d T$ (see Fig. 11a for an example). For all the sequences considered here $T_{F} \approx T_{\theta}$. For finite size systems the $\mathbf{U} \leftrightarrow \mathbf{N B A}$ transition is expected to be rounded. The rounded nature of the transition which has been seen in simulations, is reflected in the temperature dependence of $d<\chi>/ d T$ (Fig. 17a). More importantly, we expect $\Delta T / T_{F}$ to scale as

$$
\frac{\Delta T}{T_{F}} \sim N^{-1}
$$

The data for lattice Go models show that $\Delta T / T_{F} \sim N^{-\lambda}$ with $\lambda=1.2 \pm 0.1$ (Fig. 1 $\mathrm{b}$ ). The small deviation from the expected theoretical result (Eq. (5)) may be a consequence of the relatively small $N \leq 80$ in the sample. For small values of $N$ the native state does not have a well-defined core. As a result fluctuations are relatively large, which may explain the observed deviation. Analysis of the experimental data indeed shows that (Eq. (5)) is obeyed with great precision [10,33.

B: $N$ dependence of folding and unfolding barrier heights at $T_{F}$ for Go models. To compute the free energy folding barriers, $\Delta F_{F}^{\ddagger}\left(\simeq \Delta F_{U}^{\ddagger}\right.$, the unfolding barrier, at $\left.T_{F}\right)$ it is necessary to define a reaction coordinate. The precise reaction coordinate for a multidimensional process such as protein folding is difficult to ascertain. However, Onuchic and coworkers [34] have argued that, for minimally frustrated systems such as the Go models, the fraction of native contact $Q$ may be appropriate. Accordingly, we have computed $F(Q)$ for about 80 sequences with $N$ ranging from 18 to 80 . This is the largest number of sequences used so far to test the expected scaling of $\Delta F_{F}^{\ddagger}$ and $\Delta F_{U}^{\ddagger}$. At $T_{F}, \tau_{F}^{0} \exp \left(\Delta F_{F}^{\ddagger} / k_{B} T_{F}\right)=$ $\tau_{U}^{0} \exp \left(\Delta F_{U}^{\ddagger} / k_{B} T_{F}\right)$. Because it is not obvious that $\tau_{F}^{0} \approx \tau_{U}^{0}, \Delta F_{F}^{\ddagger}$ and $\Delta F_{U}^{\ddagger}$ may, in principle, exhibit different scaling behavior with $N$.

From the typical free energy profile $F(Q)$ (Fig 2 a) we computed $\Delta F_{F}^{\ddagger}$ and $\Delta F_{U}^{\ddagger}$. The variation of $\Delta F_{F}^{\ddagger} / k_{B} T$ as a function of $\ln N, N^{1 / 2}$ and $N^{2 / 3}$ for the Go sequences plotted in Fig. 2 $\mathrm{b}, \mathrm{c}, \mathrm{d}$, respectively, shows that all three fits quantitatively reproduce the simulation results. However, we argue below using the analysis of experimental data that $\Delta F_{F}^{\ddagger} \sim \ln N$ is not viable. Based on experimental estimates of $\tau_{F}^{0}$ and $\tau_{U}^{0}$ we find that $\Delta F_{F}^{\ddagger} \sim N^{1 / 2}$ provides the best physically acceptable representation of the data. From the lattice model computations we find $\Delta F_{F}^{\ddagger}$ and $\Delta F_{U}^{\ddagger}$ have the same dependence on $N$, which implies that $\tau_{F}^{0} \approx \tau_{U}^{0}$ 
C. Chain length dependence of folding rates: The RCO, which is a characteristic of the native topology of proteins, is [11]

$$
R C O=\frac{\sum_{i, j} \Delta_{i j}|i-j|}{N \sum_{i, j} \Delta_{i j}},
$$

where $|i-j|$ is the sequence separation between the residues $i$ and $j$ and $\Delta_{i j}$ is unity, if $i$ and $j$ form a native contact, or zero, otherwise. The observed correlation between $\mathrm{RCO}$ and $\ln k_{F}$ suggests that folding is most rapid, if the native state has a large fraction of local contacts. The importance of RCO is based on the sound physical idea that residues local in sequence space tend to form interactions early in the folding process and, if these substructures can "coherently" add to produce the folded structure, efficient folding may be realized. However, almost all proteins are stabilized by a sizable fraction of long-ranged (non-local) contacts. This suggests that $\ln k_{F}$ may also depend on other factors (for example, stability [20] and $N$ ) besides RCO. Lattice model simulations [35] and experiments [13] have shown that native state stability is also a contributing factor to refolding rates.

Depending on the extent of energy frustration one of us suggested [15] that $k_{F} \sim N^{\alpha}$ (for optimized sequences) or $k_{F} \sim \exp \left(-C_{1} N^{\frac{1}{2}}\right)$, where $C_{1}$ is a constant (Eqs. $\left.(1,2)\right)$. By balancing the "bulk" free energy gain due to the formation of a stable hydrophobic core and the surface tension cost due to interface formation it has been proposed [16, 17] that for optimal folding $k_{F} \sim \exp \left(-C_{2} N^{\frac{2}{3}}\right)$, where $C_{2}$ is a constant. Although, the limited range of $N$ values accessible in proteins makes it difficult to unambiguously determine the precise way $k_{F}$ decreases upon increasing $N$, it is generally agreed that free energy barriers in proteins shall be relatively small. Moreover, the transition region could be broad with roughness superimposed on it. As a result $\Delta F_{F}^{\ddagger} / k_{B} T_{F}$ is expected to grow only as $N^{\beta}$ with $\beta<1$. The sublinear growth of $\Delta F_{F}^{\ddagger} / k_{B} T$ with respect to $N$ naturally explains both the rapid folding (kinetics) and marginal stability (thermodynamics) of folded states of proteins.

Recently, Koga and Takada [36] have computed folding rates for 18 proteins using $C_{\alpha}$-Go models. They fit the data using $k_{F} \sim \exp \left(-C_{3} R C O \times N^{\beta}\right)$ with $\beta=0.607 \pm 0.179$ and $C_{3}$ is a constant. Within the error bar of their fit it is impossible to distinguish between $\beta=0.5$ or $2 / 3$. Their results showed, as argued on theoretical grounds, that $\beta<1$. In addition, due to the possibility that $\mathrm{RCO}$ decreases with $N$ [22] it is likely that the actual value of $\beta$ in [36] is considerably smaller. By focusing on the proteins that fold by three-state kinetics Galzitskaya et al. [21] have argued that chain length $N$ is the major determinant of folding rates. However, they were unable to determine the precise dependence of $k_{F}$ on $N$.

Ivankov et el. 222 have reconsidered chain length dependence of $k_{F}$ by analyzing experimental data for 57 proteins (both two and three state folders) and peptides. They suggested that $\ln k_{F} \sim-0.44 R C O \times N+11.15$ for the set of 57 proteins with the correlation coefficient $p=0.74$. For this dataset of proteins it is argued that $\mathrm{RCO} \sim N^{-0.3}$, so that $k_{F} \sim \exp \left(-C_{4} \times N^{0.7}\right)$, where $C_{4}$ is a constant. Because there are errors in fitting RCO to a power law decay with $N$, the indirect inference that $\beta \approx 0.7$ is not transparent. To circumvent this problem we have directly examined the dependence of $\ln k_{F}$ on $N$. The fit of $\ln k_{F}$ using the theoretically proposed models are shown in Fig. Ba, b and c. The correlation coefficient for the fits $\ln k_{F} \sim N^{\beta}$ is nearly constant for $0 \leq \beta \leq 2 / 3$ and begins to decrease modestly for $\beta>2 / 3$ (Fig. 3 3 d). We have also established that the folding rates in lattice Go models can be adequately fit with $\beta=0,0.5$, or $2 / 3$ [30]. From this perspective 
alone it is difficult to distinguish between the three theoretical values of $\beta(0,0.5$, and $2 / 3)$. However, we rule out $\ln k_{F} \sim \ln N$ (Fig. [3b) based on the following arguments: (1) The power law fit yields $\ln k_{F}=-5.5 \ln N+28.5$ which implies $k_{F}^{0} \approx e^{28.5} s^{-1}=(0.4 p s)^{-1}$. This value for the prefactor $k_{F}^{0}$ is nearly the same as $k_{B} T / h \approx(0.2 p s)^{-1}$, which is reasonable for small molecules, but is not appropriate to describe folding reactions. (2) The value of the exponent $\alpha=5.5$ is too large to be justified theoretically. Such a large value of $\alpha$ is usually indicative of an underlying activated process with a relatively small barrier [30].

The fits to the data in Fig. (3) cannot distinguish the scalings of $\ln k_{F}$ with $N^{1 / 2}$ or $N^{2 / 3}$. This is consistent with our results presented in Fig. 2. In an attempt to further discriminate between $\beta=0.5$ and $\beta=2 / 3$ we focus on the numerical values of the prefactor $k_{F}^{0}$. The inverse of the prefactor $1 / k_{F}^{0}$ for the $N^{1 / 2}$ scaling of the barrier height is $0.4 \mu s$, whereas $1 / k_{F}^{0} \approx 8 \mu s$ for $N^{2 / 3}$ scaling (see caption to Fig. 3). By applying Kramer's theory to describe the $\mathbf{U} \leftrightarrow$ NBA transition it has been argued that $\tau_{0}=1 / k_{F}^{0}$ should be considerably greater than $h / k_{B} T$ [37,38,39]. The range $0.4 \mu s \leq \tau_{0} \leq 8 \mu s$ obtained from the two fits is consistent with this expectation. Therefore, it follows that, unless a direct experimental measurement of $k_{F}^{0}$ is made, it would be difficult to determine the precise value of $\beta$. The goodness of fits with $\beta=1 / 2$ or $\beta=2 / 3$ shows clearly that barriers to folding scale sublinearly with $N$.

D. Prefactors for folding and unfolding. There is considerable interest in obtaining a fairly accurate estimate of $\tau_{F}^{0}\left(\sim\left(k_{F}^{0}\right)^{-1}\right)$ at near neutral $\mathrm{pH}$ and $T=25^{\circ} \mathrm{C}$ so that the measurements of average barrier heights can be made directly. Estimates of $\tau_{F}^{0}$ have been made using few physically motivated arguments:

(1) Assuming that the most elementary step in the folding process is the formation of a single tertiary contact (a loop between two residues separated by $l$ intervening residues) it was argued that the speed limit for folding is about $1 \mu s$ [40]. Because most probable loops are predicted to form in about $1 \mu s$ [42], it follows that $\tau_{F}^{0} \approx 1 \mu \mathrm{s}$. Eaton and coworkers [40,41] have provided additional arguments that proteins are unlikely to fold faster than $\tau_{F}^{0} \approx 1 \mu s$.

(2) Yang and Gruebele [43] argue using refolding data of mutants of a helical protein $\lambda_{6-85}$ that $\tau_{F}^{0} \approx 2 \mu s$. We believe that, in general, for the majority of proteins $\tau_{F}^{0} \approx 2 \mu s$ should be near the upper limit for the following reasons. Based on theories of collapse dynamics we expect that the 80 residue protein $\lambda_{6-85}$ becomes compact in about $\tau_{c} \approx(\eta a / \gamma) N^{\theta} \approx 1.5 \mu s$, where $\eta$ is the solvent viscosity, $a$ is the Flory characteristic ratio, $\gamma$ is the surface tension (50 cal $\left./ \mathrm{mol} \AA^{2}\right), \theta \approx 2.2$ and $N=80$. This estimate is close to the folding time for $\lambda_{6-85}$, which suggests that collapse and folding are nearly simultaneous for this protein. Because these two processes cannot be separated for proteins with $N=80$ that fold in about $\approx 1 \mu \mathrm{s}$, it appears that one can assume that $\tau_{F}^{0} \approx 2 \mu s$ may be an upper bound. We believe that $\tau_{F}^{0} \approx 1 \mu s$ could serve as a practical estimate for the prefactor, because on time scales greater than $1 \mu s$ multiple loops can form and collapse of the entire polypeptide chain can occur, which could obfuscate direct determination of $\tau_{F}^{0}$. In arriving at these estimates for $\lambda_{6-85}$ we have assumed that internal viscosity does not alter folding rates appreciably. Although, a similar observation has been made for refolding of protein L [44] and CspB [45] it is unclear how important internal viscosity of proteins is in the determination of $\tau_{F}^{0}$ [39]. In addition, external conditions can alter $\tau_{F}^{0}$. Thus, $\tau_{F}^{0} \approx 1 \mu s$ should be taken merely as a useful estimate for the prefactor.

The dependence of $\ln k_{F}$ on $N$ (Fig. (3)) allows us to estimate $\tau_{F}^{0}$ and $\tau_{U}^{0}$ using the 
experimental data for proteins that are not fully represented in Fig. (3). We use refolding rates for several $\beta$-sheet proteins to estimate $\tau_{F}^{0}$ (Table 1). Assuming $N^{1 / 2}$ scaling we find that $\tau_{F}^{0}$ is in the range $(0.1-18) \mu s$. Except for $\tau_{F}^{0} \simeq 18 \mu s$ obtained for twitchin $\left(\operatorname{TWIg} 18^{\prime}\right)$ with low native state stability the average value of the prefactor is $\overline{\tau_{F}^{0}} \approx 3.5 \mu \mathrm{s}$. If we use $\tau_{F}^{0} \simeq \tau_{F} \exp \left(-0.36 N^{\frac{2}{3}}\right.$ ) (Fig. (3)), we find $2 \mu s \lesssim \tau_{F}^{0} \lesssim 400 \mu s$ (Table 1). For four immunoglobulin proteins with the exception of FNfn10 (Table 1) the estimated values of $\tau_{F}^{0}$ using the $N^{\frac{2}{3}}$ scaling for the barrier height seem too large. Thus, $\tau_{F}^{0}$ appears to be in the neighborhood of few $\mu s$ for the $\beta$-sheet proteins and for the $\alpha$-helical protein $\lambda_{6-85}$.

Another question of interest is whether $\tau_{F}^{0} \approx \tau_{U}^{0}$ ? Using lattice model simulations we have previously argued that the unfolding and folding prefactors are similar [35]. This conclusion was reached using the number of native contacts $Q$ as a reaction coordinate. It is unclear whether this result is a consequence of our choice of the reaction coordinate. The results in Fig. (3) and the measured unfolding rates in Table 1 allow us to directly estimate

$$
\tau_{U}^{0} \simeq \tau_{U} \exp \left[-\left(1.1 N^{1 / 2}+\beta \Delta G\right)\right]
$$

where $\tau_{U}$ is the unfolding time, $\Delta G$ is the free energy of stability of the native state, and $\beta=\left(k_{B} T\right)^{-1}$. With the exception of TWIg18' the ratio $\tau_{U}^{0} / \tau_{F}^{0}<1$ and is in the range $0.1 \lesssim \tau_{U}^{0} / \tau_{F}^{0} \lesssim 1.0$. For this class of proteins the maximum value of $\tau_{F}^{0} / \tau_{U}^{0} \lesssim 10$ (Table 1 ). Similar conclusions have been drawn for $\alpha$-helical proteins as well. Thus, it appears that $\tau_{U}^{0} \approx \tau_{F}^{0}$.

\section{CONCLUSIONS}

In this article we have considered finite size effects in thermal denaturation and folding kinetics. We have established using lattice models that the rounded transition as quantified by $\Delta T / T_{F}$ obeys the expected scaling (Eq. (5)). This is in accord with the earlier analysis of the experimental data [10], which further suggests that qualitative features of folding transition can be gleaned using lattice models. Unlike the case of thermal denaturation the situation is far more ambiguous when the scaling of $k_{F}$ with $N$ is considered. The dependence of $\ln k_{F}$ on $N$ does not match the quality of correlation noted for thermodynamics. If we delete the fastest folding proteins and peptides and the slowest folding proteins from the dataset in Fig. 3, the correlation coefficient becomes considerably worse $(\approx 0.56)$ regardless of the scaling ( $\beta=0.5$ or $2 / 3)$ used. Nevertheless, the inclusion of the $N$ dependence does improve the correlation between $\ln k_{F}$ on RCO [22]. Using the expected values (from a number of unrelated studies) for the prefactor, we suggest that the $N^{1 / 2}$ scaling for barrier height $\Delta F^{\ddagger} / k_{B} T$ may be useful in making order of magnitude estimates of refolding rates. This scaling also implies that the energy landscape of two-state proteins is rugged. The energy scale for roughness may be of order of a few $k_{B} T$.

We are grateful to A. Finkelstein for permission to use the data in Ref. [22] and for useful discussions. We are pleased to acknowledge conversations with M. Gruebele, V. Munoz and W. A. Eaton on the experimental results of the determination of folding prefactors. Mai Suan Li would like to thank the hospitality of IPST, where part of this work has been done. This work was supported in part by a KBN grant and the National Science Foundation grant (NSF CHE-0209340). 


\section{REFERENCES}

[1] Onuchic J N, Luthey-Schulten Z, and Wolynes P G. Ann Rev Phys Chem 1997; 48: 545-600.

[2] Thirumalai D and Klimov D K. Curr Opin Struct Biol 1999; 9: 197-207.

[3] Dill K A and Chan H S. Nat Struc Biol 1997; 4: 10-19.

[4] Mirny L and Shakhnovich E. Ann Rev Biophys Biom 2001; 30: 361-396.

[5] Bonneau R and Baker D. Ann Rev Biophys Biomol Struct 2001; 30: 173-190.

[6] Klimov D K and Thirumalai D. Proc. Natl. Acad. Sci. USA 2000; 97: 2544-2549.

[7] Munoz V, Thompson P A, Hofrichter J and Eaton W A. Nature 1997; 390: 196-199.

[8] Lakshmikanth G S, Sridevi K, Krishnamoorthy G, and Udgaonkar J B. Natur Struct Biol 2001; 8: 799-804.

[9] Holtzer M E, Lovett E G, d'Avignon D A, and Holtzer A. Biophys J 1997; 73: 1031-1041.

[10] Klimov D K and Thirumalai D. J Comp Chem 2002; 23: 161-165.

[11] Plaxco K W, Simons K T, and Baker D. J Mol Biol 1998; 277: 985-994.

[12] Baldwin R L and Rose G. Trends Biochem Sci 1999; 24: 77-83.

[13] Clarke J R, Cota E, Fowler S B and Hamill S J. Structure 1999; 7: 1145-1153.

[14] Scott K A, Steward A, Fowker S B and Clarke J. J. Mol. Biol. 2002; 315: 819-829.

[15] Thirumalai D. J Phys I (France) 1995; 5: 1457-1467.

[16] Wolynes P G. Proc. Natl. Acad. Sci. USA 1997; 94: 6170-6175.

[17] Finkelstein A V and Badretdinov A Ya. Fold Des 1997; 2: 115-121.

[18] Gutin A M, Abkevich V I, and Shakhnovich E I. Phys Rev Lett 1996; 77: 5433-5436.

[19] Perl D, Welker C, Schindler T, Schroder K, Marahiel M A, Jaenicke R and Schmid F X. Nat Struct Biol 1998; 5: 229-235.

[20] Dinner A R and Karplus M. Nat Struct Biol 2001; 8: 21-22.

[21] Galzitskaya O V, Garbuzynskiy S O, Ivankov D N, and Finkelstein A V. Proteins Struct Funct Cenet 2003; 51: 162-166.

[22] Ivankov D N, Garbuzynskiy S O, Alm E, Plaxco K W, Baker D and Finkelstein A V. Protein 2003; 12: 2057-2062.

[23] Camacho C J and Thirumalai D. Proc. Natl. Acad. Sci. USA 1993; 90: 6369-6372.

[24] Cieplak M , Hoang T X, and Li M S. Phys Rev Lett 1999; 83: 1684-1687.

[25] Kaya H and Chan H S. J Mol Biol 2002; 315: 899-909.

[26] Kaya H and Chan H S. J Mol Biol 2003; 326: 911-931.

[27] Betancourt M R. J Chem Phys 1998; 109: 1545-1554.

[28] Betancourt M R and Thirumalai D. J Phys Chem B 2002; 106: 599-609.

[29] Li M S, Klimov D K and Thirumalai D. Comp Phys Commun 2002; 147: 625-628.

[30] Li M S, Klimov D K and Thirumalai D. J Phys Chem B 2002; 106: 8302-8305.

[31] Hilhorst H J and Deutch J M. J Chem Phys 1975; 63: 5153-5161.

[32] Ferrenberg A M and Swendsen R H. Phys Rev Lett 1989; 63: 1195-1198.

[33] Li M S, Klimov D K and Thirumalai D. (unpublished results).

[34] Nymeyer H, Garcia A E, Onuchic J N. Proc. Natl. Acad. Sci. USA 1998; 95: 5921-5926.

[35] Klimov D K and Thirumalai D. J Chem Phys 1998; 109: 4119-4125.

[36] Koga N and Takada S. J Mol Biol 2001; 313: 171-180.

[37] Klimov D K, Thirumalai D. Phys. Rev. Lett. 1997; 79: 317-320.

[38] Socci N D, Onuchic J N, Wolynes P G. J. Chem. Phys. 1996; 104:5860-5868.

[39] Portman J J, Takada S, Wolynes P G. J Chem Phys 2001; 114: 5069-5081. 
[40] Hagen S J, Hofrichter J, Szabo A and Eaton A W. Proc Natl Acad Sci USA 1996; 93: 11615-11619.

[41] Munoz V and Eaton W A. Proc Natl Acad Sci USA 1999; 96: 11311-11316.

[42] Guo Z and Thirumalai D. Biopolymers 1995; 36: 83-102.

[43] Yang W Y and Gruebele M. Nature 2003; 423: 193-197.

[44] Plaxco K W, Millett I S, Segel D J, Doniach S and Baker D. Nat Struc Biol 1999; 6: 554-556.

[45] Jacob M, Schindler T, Balbach J, Schmid F X. Proc Natl Acad Sci USA 1997; 94:56225627. 
Table 1. Estimates of the folding and unfolding prefactors ${ }^{a}$

\begin{tabular}{lrrrrrrr}
\hline protein $^{b}$ & $\beta \Delta G^{c}$ & $\tau_{F}^{d}$ & $\tau_{U}^{e}$ & $\tau_{F}^{0 f}$ & $\tau_{F}^{0 g}$ & $\tau_{U}^{0 h}$ & $\tau_{U}^{0 i}$ \\
\hline TI I27 (89) & 12.7 & 0.0313 & 2041 & 0.974 & 23.9 & 0.194 & 4.76 \\
TWIg18'(93) & 6.9 & 0.667 & 3571 & 16.5 & 412 & 89.0 & 2220 \\
CD2d1(98) & 11.5 & 0.0556 & 588 & 1.04 & 26.4 & 0.111 & 2.83 \\
TNfn3 (92) & 9.1 & 0.344 & 2174 & 9.00 & 224 & 6.35 & 158 \\
FNfn10 (96) & 15.9 & 0.00417 & 4348 & 0.0870 & 2.20 & 0.0113 & 0.285 \\
CspB (B.subtilis)(67) & 4.6 & 0.00145 & 0.101 & 0.178 & 3.82 & 0.125 & 2.68 \\
CspB (B.caldolyticus)(66) & 8.1 & 0.000730 & 1.56 & 0.0960 & 2.04 & 0.0623 & 1.32 \\
CspB (T.maritima)(68) & 10.6 & 0.00177 & 55.6 & 0.203 & 4.40 & 0.159 & 3.44 \\
\hline
\end{tabular}

(a) Data for the first five proteins are from [13] and the data for CspB proteins are from [19]

(b) Numbers in parenthesis are the values of $N$

(c) Free energy of stability extrapolated to zero denaturant concentration

(d) Folding times in seconds

(e) Unfolding times in seconds

(f) Folding prefactor (in units of $\mu s$ ) calculated using $\tau_{F}^{0}=\tau_{F} \exp \left(-1.1 N^{1 / 2}\right)$

(g) Folding prefactor (in units of $\mu s$ ) calculated using $\tau_{F}^{0}=\tau_{F} \exp \left(-0.36 N^{2 / 3}\right)$

(h) Unfolding prefactor (in $\mu s$ ) calculated using $\tau_{U}^{0}=\tau_{U} \exp \left(-1.1 N^{1 / 2}-\Delta G /\left(k_{B} T\right)\right)$

(i) Unfolding prefactor (in $\mu s$ ) calculated using $\tau_{U}^{0}=\tau_{U} \exp \left(-0.36 N^{2 / 3}-\Delta G /\left(k_{B} T\right)\right.$ ) 


\section{Figure captions}

Fig. (1) (a) Temperature dependence of $d\langle\chi\rangle / d T$ for the lattice sequence with $N=64$. The folding transition temperature is identified with the peak in $d\langle\chi\rangle / d T$. The full width at half-maximum is indicated by $\Delta T$. (b) The dependence of $\Delta T / T_{F}$ as a function of $N$. The straight line gives the fit $\Delta T / T_{F} \sim N^{\lambda}$ with $\lambda=1.2 \pm 0.1$.

Fig. (2) (a) Dependence of $F / k_{B} T$ ( $F$ is the free energy of a sequence) as a function of the presumed reaction coordinate $Q$, the number of native contacts, for one of the $N=64$ Go sequences. The unfolding and refolding barriers are extracted from the free energy profile as indicated. Panel (b) shows the fit $\Delta F_{F}^{\ddagger} / k_{B} T_{F} \sim \ln N$. Panels (c) and (d) correspond to the fits $\Delta F_{F}^{\ddagger} / k_{B} T_{F} \sim N^{\beta}$ with $\beta=0.5$ and $2 / 3$, respectively. The results were computed for $N=18(20), 27(17), 36(18), 48(18), 64(15)$, and 80(12), where the number in parenthesis refers to the number of sequences used for averaging $\Delta F_{F}^{\ddagger} / k_{B} T_{F}$. Similar scaling with $N$ is obtained for $\Delta F_{U}^{\ddagger} / k_{B} T_{F}$.

Fig. (3) Fits of $\ln k_{F}$ as a function of $N$ for the dataset of 57 proteins and peptides taken from ref. [22]. Cross and hexagon symbols correspond to three and two state folders, respectively. (a) The fit based on $\ln k_{F} \sim N^{\frac{1}{2}}$. The straight line is $y=-1.1 x+14.7$ and the correlation coefficient is 0.71 . (b) The fit based on $\ln k_{F} \sim N^{\frac{2}{3}}$. The straight line is $y=-0.36 x+11.7$ and the correlation coefficient is 0.70 . (c) Fits of $\ln k_{F} \sim \ln N$ gives $y=-5.5 x+28.5$ with the correlation coefficient of 0.72 . (d) Variation of the correlation coefficient with $\beta$. The correlation becomes weaker at $\beta>2 / 3$. 


\section{FIGURES}

(a)

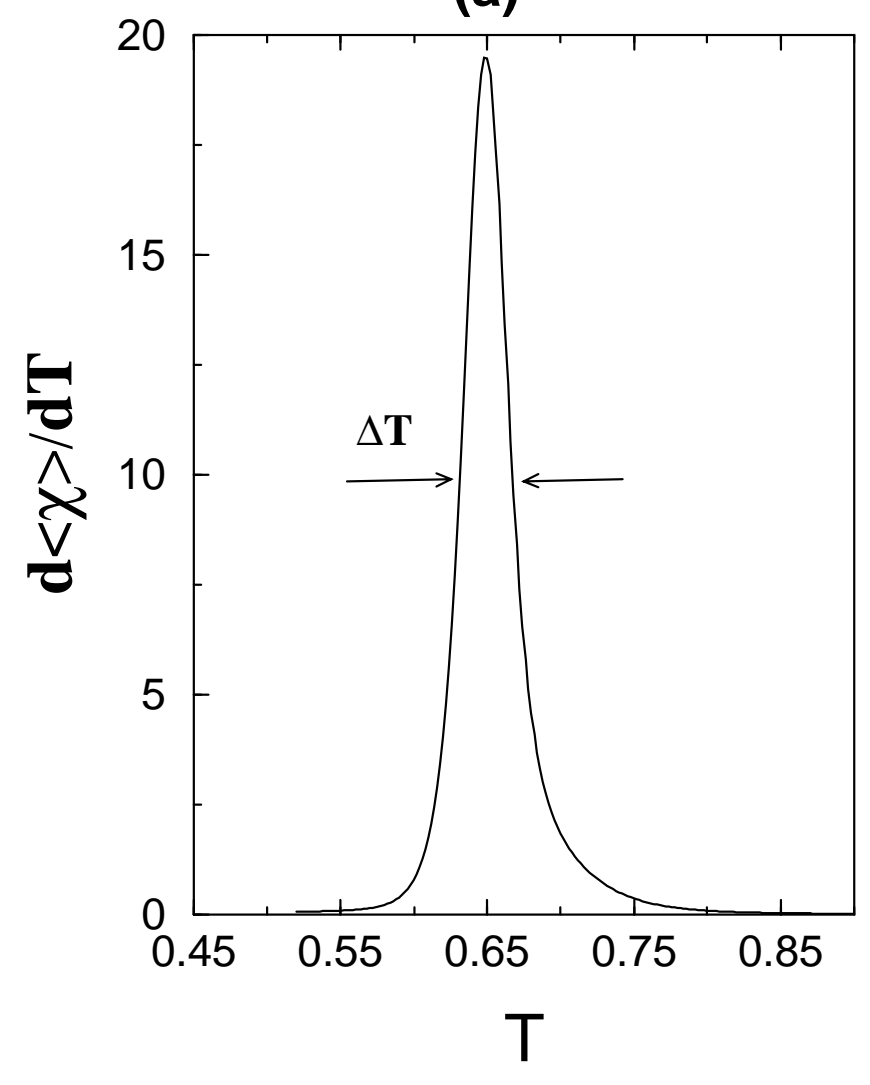

(b)

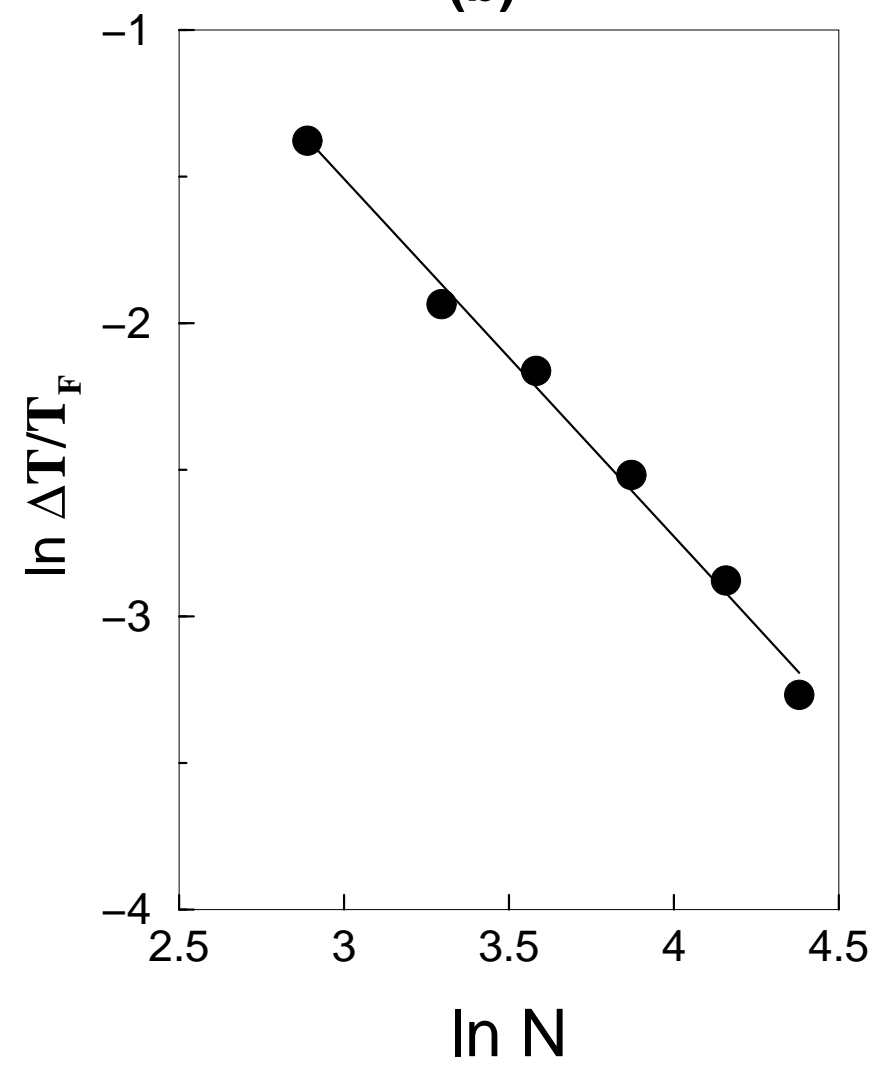

FIG. 1. 

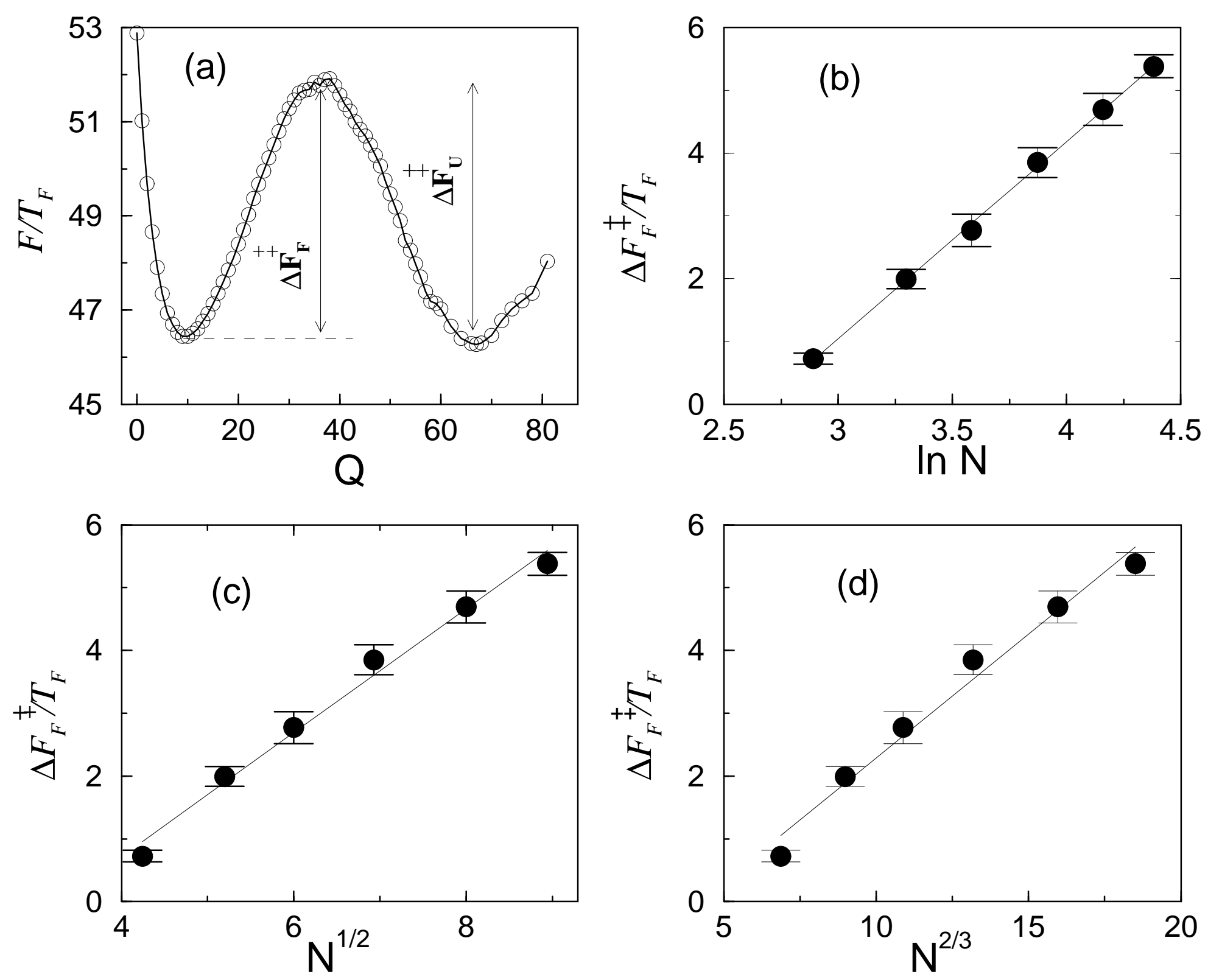

FIG. 2. 

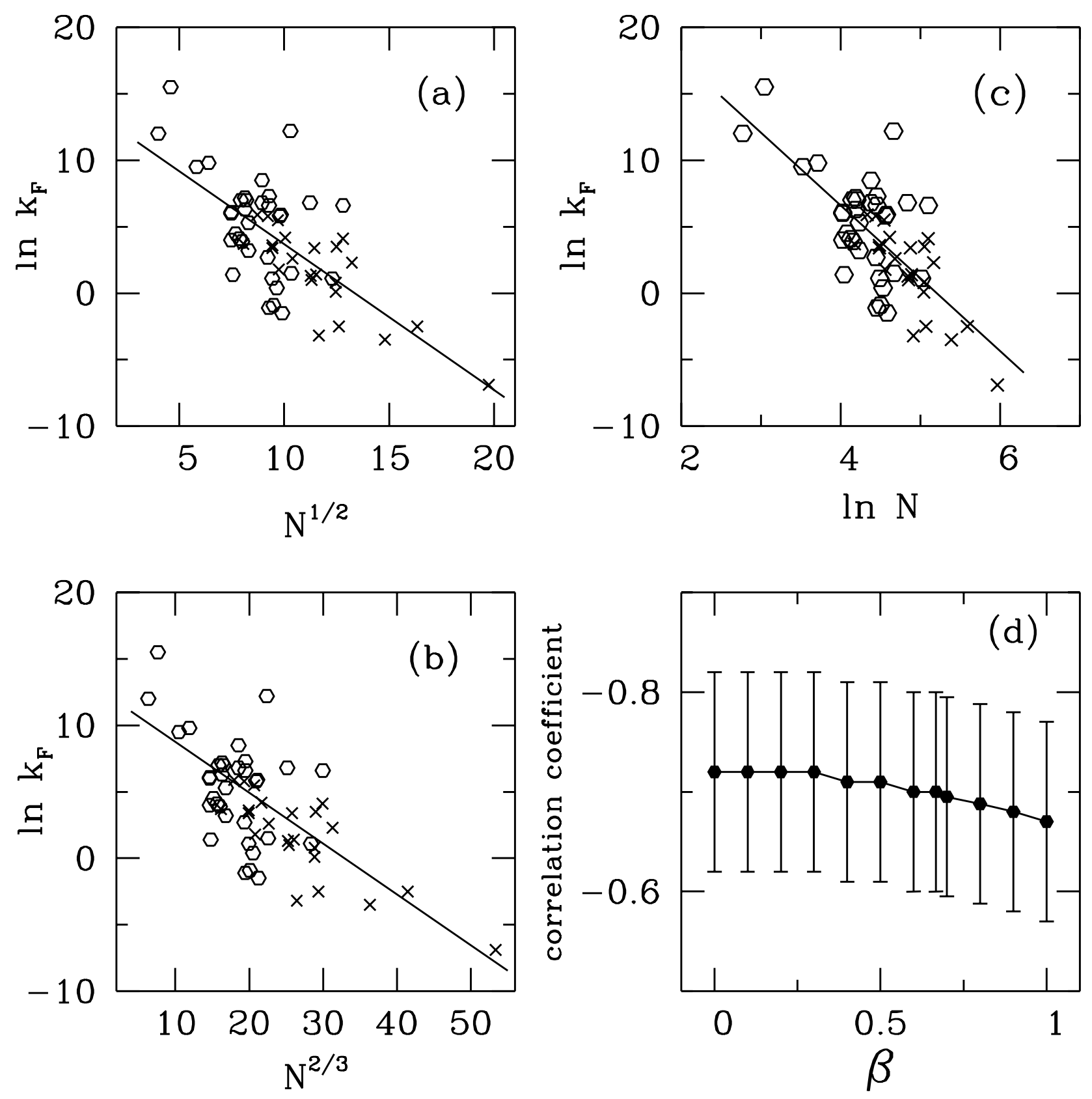

FIG. 3. 